\title{
Language difficulties in children with Attention Deficit Hyperactivity Disorder
}

Paraskevi Agapitou ${ }^{1}$, Ioannis Galantomos ${ }^{2}$, Georgia Andreou ${ }^{3}$

${ }^{1}$ Department of Special Education, University of Thessaly, Greece

${ }^{2}$ Department of Special Education \& Department of Language, Literature and

Culture of the Black Sea Countries, University of Thessaly \& Democritus

University of Thrace, Greece

${ }^{3}$ Department of Special Education, University of Thessaly, Greece

https://doi.org/10.36505/ExLing-2010/03/0001/000121

\begin{abstract}
In this study we review the relevant literature regarding the connection of Attention deficit hyperactivity disorder (ADHD) with language disorders. Special emphasis is given to the evolution of language disorders in relation with ADHD, because both of them appear during preschool age and influence in a crucial manner the acquisition of basic learning readiness skills. The review of a considerable body of literature indicates that the co morbidity of ADHD with language difficulties worsens children's behaviour problems at preschool age and affects their socialization and academic performance. Hence, it is concluded that a proper and valid diagnosis of ADHD and language difficulties plus combined and suitable interventions will reduce the amount and quality of ADHD symptoms.
\end{abstract}

Key words: ADHD, language difficulties, behaviour

\section{Introduction}

ADHD is the most common psychiatric disorder in childhood, affecting approximately $3-7 \%$ of school-age children. This disorder has specific subtypes and exhibits differences in children who display predominantly inattentive behaviours, hyperactive and impulsive behaviour or a combination of both (Barkley 1998; APA 2000). Although there has been a number of studies describing the relationship between language disorders and behaviour disorders in children there have been relatively few data available that directly examine the language skills of children with a particular subtype of behaviour disorder: Four possible theoretical models have been proposed: (a) behaviour problems are a consequence of children's frustration with their reading difficulties; (b) behaviour problems are a cause of reading difficulties; (c) behaviour problems and reading difficulties are neither a cause nor a consequence but share a common cause; or (d) all of the proposed relationships could be active in some sense, with bi-directional or transactional influences best characterizing the relationship between behaviour and achievement (Spira \& Fischer 2005).

ExLing 2010: Proceedings of $3^{\text {rd }}$ Tutorial and Research Workshop on Experimental Linguistics, 25-27 August, Athens, Greece 


\section{Comorbidity}

Language impairments are commonly observed among children referred for psychiatric services. The most frequent psychiatric diagnosis of children with language impairment (LI) is ADHD. It is not clear whether there are differences between children with ADHD and comorbid LI and children with other psychiatric disorders who are also comorbid for LI. Developmental disorders might differ in their language profiles when using parent reports (Geurts et al. 2008).

Many of the studies of the language abilities of children with ADHD have not yet distinguished between children with ADHD alone and those with comorbid disorders. Thus, it is difficult to determine whether the language impairments are specific to ADHD or whether they are more a corollary of the comorbid disorder (Purvis \& Tannock 1997). Cohen et al. (2000) examined four groups: children with ADHD-LI, children with ADHD who have normally developing language, children with psychiatric diagnoses other than ADHD with language impairment or without LI. Results indicated that children with LI were the most disadvantaged regardless of the nature of the psychiatric diagnosis.

\section{Language and academic deficits}

It is clear that some children with symptoms of ADHD at preschool age are at risk for long-lasting behavioural difficulties. Additional research suggests that children who manifest behavioural difficulties associated with ADHD at preschool age may be at risk for academic underachievement as well. There is a large amount of literature on the relationship between learning problems and ADHD in elementary-school-aged children. For instance, Agapitou \& Andreou (2008) found that ADHD preschoolers who demonstrate serious language deficits are more liable to develop poor reading skills in primary school.

Children diagnosed with Attention Deficit Hyperactivity Disorder (ADHD) have cognitive processing difficulties due to their disinhibition and attention deficits, which influence their scholastic performance. Cognitive processing has also an impact on the production of oral narratives, an essential skill required for academic success. According to Moonsamy et al. (2009), narrative measures are useful instruments for oral language evaluation in children with ADHD and the importance of understanding oral narratives within a therapeutic situation is important for both therapist and child. 
Thus, problems with language and pragmatics seem to be associated with the typical problems with learning and social skills in children with ADHD. Children and adolescents with ADHD are slower and less efficient than matched control subjects with regard to complex sentence comprehension (Wassenberg et al. 2010).

Children with ADHD use fewer strategies of textual organization and more avoidance, tangential, and unrelated meanings and more abandoned utterances and spelling and punctuation errors (Mathers 2006).

\section{Conclusion}

To sum up, the above mentioned review of a considerable body of recent literature suggests that ADHD along with difficulties manifested in language affect children's behaviour at preschool age in a negative manner. Furthermore, the combination of ADHD with language problems has an impact on children's overall academic performance and socialization skills. Therefore, it is concluded that a valid and in time diagnosis of both ADHD and language problems combined with a multifaceted intervention will reduce the amount of evidenced symptoms and ameliorate children's quality of life.

\section{References}

Agapitou, P., Andreou, G. 2008. Language deficits in ADHD preschoolers. Australian Journal of Learning Difficulties 13, 39-49.

American Psychiatric Association (APA). 2000. Diagnostic and statistical manual of mental disorders - 4th edition, text revision (DSM-IV-TR). Arlington, VA: APA.

Barkley, R.A. 1998. Attention deficit hyperactivity disorder: A handbook for diagnosis and treatment. New York, Guilford Press.

Cohen, N.J., Vallance, D.D., Barwick, M., Im, N., Menna, R., Horodezky, N.B. 2000. The interface between ADHD and language impairment: An examination of language, achievement, and cognitive processing. Journal of Child Psychology and Psychiatry 41, 353-362.

Geurts, H., Embrechts, M. 2008. Language Profiles in ASD, SLI, and ADHD. Journal of Autism and Developmental Disorders 38, 1931-1943

Mathers, M. 2006. Aspects of Language in Children with ADHD. Applying Functional Analyses to Explore Language Use. Journal of Attention Disorders 9, 523-533.

Moonsamy, S. Jordaan, H., Greenop, K. 2009. Cognitive processing and narrative discourse production in children with ADHD, South African Journal of Psychology 39, 326-335.

Purvis, K.L., Tannock, R. 1997. Language abilities in children with ADHD, reading disabilities and normal controls. Journal of Abnormal Child Psychology 25, 133144. 
Spira, E.G., Fischer, J.E. 2005. The impact of preschool inattention, hyperactivity, and impulsivity on social and academic development: a review. Journal of Child Psychology and Psychiatry 46, 755-773.

Wassenberg, R., Hendriksen, J., Hurks, P., Feron, F., Vles, J., Jolles, J. 2010. Speed of Language Comprehension is Impaired in ADHD. Journal of Attention Disorder $13,374-385$. 